\title{
IMAGERIE MULTISPECTRALE PAR FILTRE DE BAYER CUSTOMISÉ : émergence d'une technologie intégrée
}

\author{
Stéphane TISSERAND \\ SILIOS Technologies \\ stephane.tisserand@silios.fr
}

\begin{abstract}
La spectroscopie, science fondée par Isaac Newton en 1666, qui traite de l'interaction des différents types de rayonnements avec la matière, a donnée naissance à la spectrométrie qui concerne plus précisément l'instrumentation et les mesures en vue d'étude spectroscopique. Développée au cours des trois siècles qui ont suivi jusqu'à aujourd'hui, cette dernière a produit de nombreux instruments, de plus en plus sophistiqués permettant la mesure des spectres d'absorption, d'émission et de diffusion de la matière soumise aux rayonnements électromagnétiques.
\end{abstract}

\begin{abstract}
T) jents analyse des signatures spectrales de matériaux ou d'objets est aujourd'hui utilisée dans un nombre très vaste de domaines scientifiques ou industriels pour mesurer ou simplement détecter des constituants. Côté science, l'astronomie au sol en premier lieu a su développer très tôt des instruments performants permettant d'accéder à une grande finesse dans la mesure spectrale des astres, galaxies et autres corps célestes. Dans l'industrie, le domaine de la chimie dans ses multiples branches a également rapidement cherché à exploiter la spectrométrie et a développé un large savoir-faire.

Malgré tout, un spectromètre ne permet de mesurer le spectre d'un objet qu'en un point. La mesure peut être éventuellement réalisée sur une zone étendue (voire sur un objet complet) mais elle ne fournira alors qu'un spectre moyen de la zone concernée. Or dans un grand nombre de cas, on cherche soit à qualifier l'uniformité del'objet, soità discriminer différentes régions d'un objet ou d'une scène. Il est alors nécessaire d'effectuer de multiples mesures échantillonnées spatialement (ou la scène), ce qui n'est pas toujours possible pour des questions de cinétique (évolution de l'objet au cours de la mesure) ou simplement de durée de la mesure (durée de mesure prohibitive ou inadaptée au contexte comme dans le cas des applications embarquées par exemple).
\end{abstract}

\section{Imagerie hyperspectralle et multispectirale}

Les notions d'imagerie hyperspectrale et multispectrale sont issues de ce besoin de multiplexage spatial de l'information spectrale. Un imageur hyper/multispectral permet d'enregistrer une image 2D tout en extrayant le spectre de chaque point de l'image. L'ensemble de ces données spatiales et spectrales est communément appelé un «hypercube». En règle générale, on parle d'imagerie hyperspectrale lorsque les spectres extraits sont très résolus, c'est-à-dire des spectres dont le nombre de points d'échantillonnage est supérieur à 100 , et d'imagerie multispectrale lorsque les spectres comportent moins de 20 points d'échantillonnage.

Historiquement, une fois encore, c'est le domaine de l'astronomie et du spatial, qui pour des applications de télédétection, a développé les premiers systèmes d'imagerie multispectrale. Ces systèmes utilisés pour l'observation de la Terre ou d'autres planètes sont aujourd'hui essentiellement basés sur l'association d'un plan image avec un ensemble discret de filtres de géométrie rectangulaire très allongés, dits filtres allumettes. Ces filtres couvrent l'imageur sur quelques pixels dans une direction et l'ensemble des pixels dans l'autre direction. L'imageur multispectral enregistre ainsi plusieurs bandes spectrales juxtaposées. Le mouvement du satellite permet d'obtenir, par balayage, l'information spatiale 2D complète. Ces informations multispectrales sont largement exploitées dans le domaine de l'environnement et de l'agriculture depuis des dizaines d'années. Dans le domaine de la surveillance aérienne, des systèmes hyperspectraux ont également vu le jour, souvent basés sur l'utilisation d'un réseau de diffraction couplé à un imageur. Le réseau disperse le spectre dans une direction du plan de l'imageur. L'image 2D cette fois encore est reconstruite grâce au déplacement de l'appareil. La télédétection spatiale et la surveillance aéroportée sont restées longtemps les deux seuls domaines à tirer parti du multispectral. Les instruments utilisés pour ces applications sont chers, souvent encombrants et contraignants à utiliser. Ils ne peuvent que difficilement être réutilisés dans d'autres domaines. 


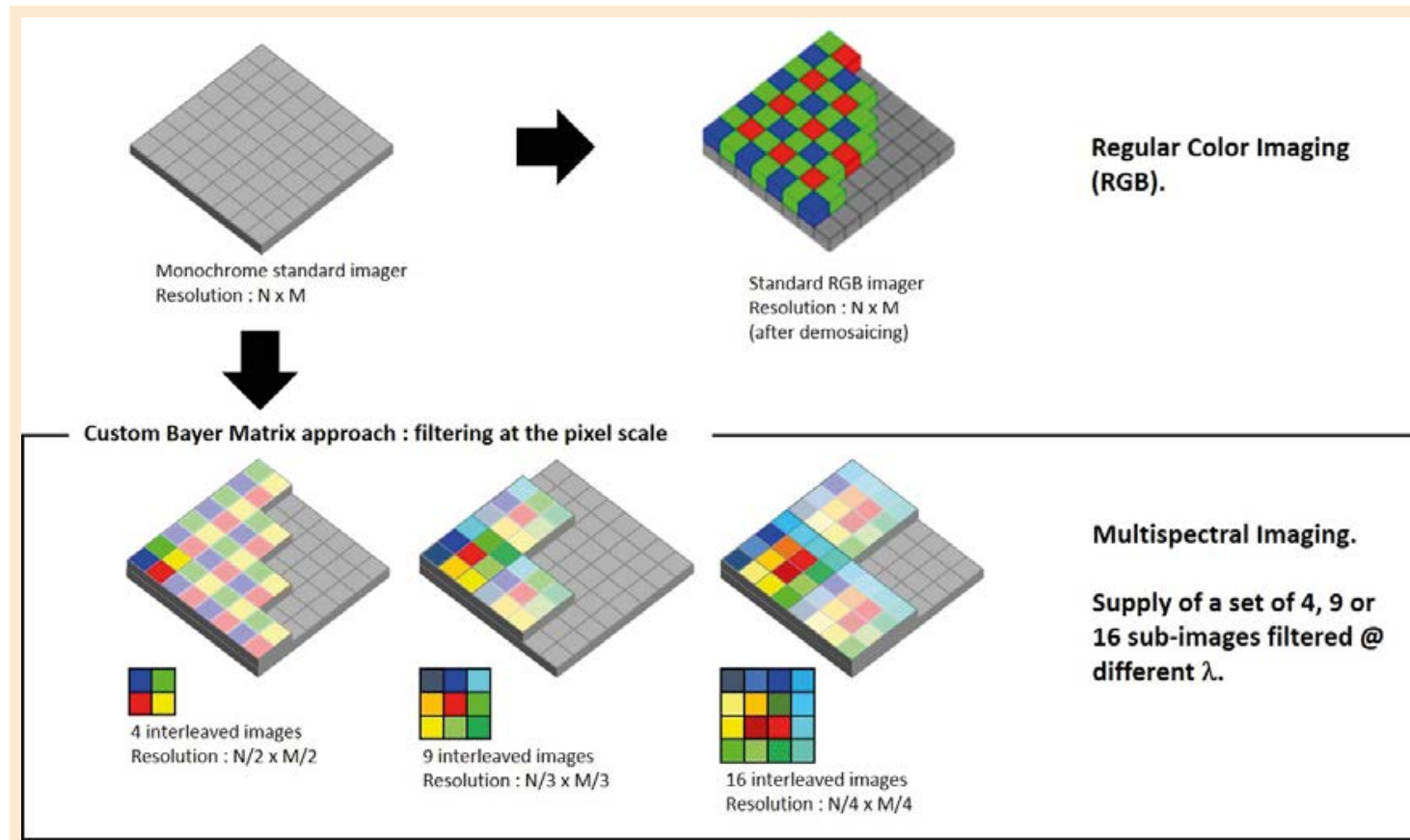

Figure 1. Principe de la matrice de Bayer sur mesure pour l'imagerie multispectrale.

D'autres systèmes multispectraux plus rudimentaires, basés sur des roues à filtres, ont été développés principalement pour des applications industrielles fixes (en laboratoires, sur chaîne de fabrication...), mais également pour des applications de surveillance au sol (détection de fuites de gaz par exemple).

Ces systèmes associent un imageur et des filtres interférentiels montés sur une roue qui viennent successivement se positionner devant l'imageur. Ce type de système présente plusieurs inconvénients. Son encombrement est incompressible, sa fiabilité et sa robustesse sont limités par l'existence de parties mobiles. D'autre part, la mesure est séquentielle (non synchrone) ce qui interdit la mesure embarquée ou la mesure de scènes ou d'objets dont la dynamique est supérieure au temps de mesure complet (passage de tous les filtres devant l'imageur).

Enfin, pour les applications ne nécessitant qu'un très faible échantillonnage spectral (typiquement moins de 5 bandes), certains systèmes intègrent autant d'imageurs que de bandes spectrales à mesurer, un filtre spécifique venant «coiffer» chaque imageur. La difficulté consiste alors à aligner optiquement les différents capteurs pour obtenir un point de vue identique dans chaque bande spectrale (en cadrage et angulairement) ou à recombiner les différentes images spectrales par logiciel. Le faible nombre de bandes accessibles reste une limitation pour beaucoup d'applications.

Tous les systèmes présentés ci-dessus (roues à filtres, réseaux de diffraction, multi-imageurs) présentent par ailleurs

Figure 2. Caméras multispectrales à filtres pixélisés matriciel proposées par la société SILIOS Technologies.

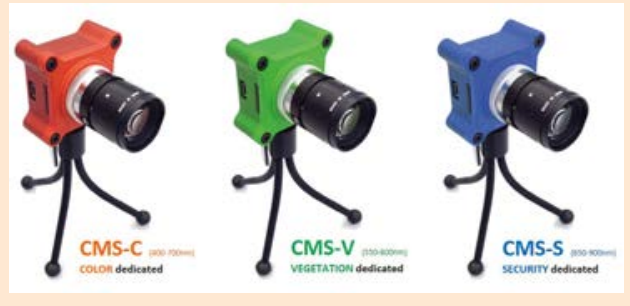

un inconvénient majeur inhérent à tout instrument optique complexe: la fiabilité et la robustesse vis-à-vis des contraintes mécaniques et thermiques.

\section{Applications émergentes}

En terme applicatif, de nouveaux domaines s'intéressent de près à l'imagerie hyper/multispectrale. L'essor de l'économie numérique et de l'Internet des objets, pour répondre à de nombreux défis sociétaux (santé, sécurité, efficience énergétique, alimentation, surveillance de l'environnement...), pousse ainsi différents secteurs à s'intéresser à ces imageurs d'un nouveau genre. L'agriculture moderne ou agriculture de précision par exemple subit aujourd'hui une transformation importante avec une introduction marquée de la robotique dans la gestion de ses procédés. Un nombre grandissant de projets voient le jour pour l'automatisation de la production (usines pilotes de production maraichère automatisée) et l'optimisation des cultures. L'utilisation de drones volants embarquant des systèmes multispectraux à 4 ou 5 bandes se déploie pour la cartographie des champs de grandes cultures et l'optimisation de la dispense des fertilisants. Les drones terrestres sont également en plein développement pour le désherbage ou le traitement de certaines maladies. Le domaine médical, et plus particulièrement la dermatologie et la cancérologie, testent également les solutions multispectrales afin de déterminer leur potentiel à détecter de manière plus précoce certaines pathologies. De la même manière, l'industrie cosmétique commence à exploiter l'imagerie multispectrale dans le contexte en plein essor de la personnalisation des soins. L'analyse de l'image multispectrale de la peau d'un visage par exemple peut être exploitée pour la préconisation d'un soin sur mesure. Enfin, la capacité de segmentation de scènes que permet l'imagerie multispectrale est un sujet d'étude pour le développement et la fiabilisation des véhicules autonomes. 


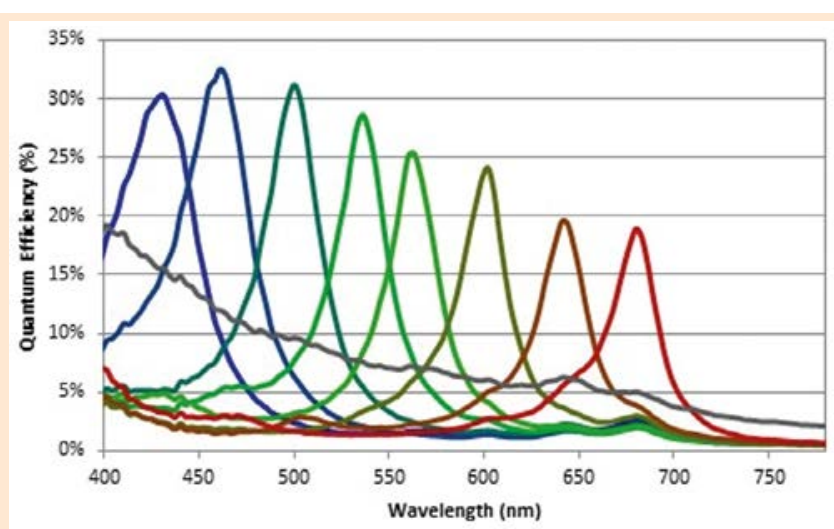

Figure 3. Rendement quantique $\mathrm{QE}$ des différents pixels de l'imageur multispectral CMS-C.

Pour la plupart, ces applications recherchent des solutions technologiques de caméras multispectrales présentant les caractéristiques suivantes:

- forte compacité,

- faible poids,

- nombre de bandes supérieur à 5 ou 6 ,

- système synchrone (idéalement: une image intégrant toute l'information spectrale),

- robustesse et fiabilité,

- bas coût.

\section{Approche par filltre de Bayer customisée}

Une approche alternative aux systèmes précédemment décrits consisteàétendre le principe de la matrice de Bayer utilisé communément pour enregistrer les images numériques en couleur (figure 1). La matrice de Bayer standard déposée sur un imageur pixélisé monochrome, est constituée de macro-pixels de $2 \times 2$ pixels ${ }^{2}$, répliqué matriciellement sur toute la surface de l'imageur. Ce macro-pixel est lui-même composés d'un pixel rouge, d'un pixel bleu et de deux pixels verts. La réalisation de filtres interférentiels diélectriques passe-bandes par procédés de micro-technologie (photolithographie, dépôt de couches minces, gravures...) permet de définir des macro-pixels sur mesure de $2 \times 2,3 \times 3$ ou $4 \times 4$ pixels ${ }^{2}$.

La réalisation de tels filtres pixélisés matriciels et leur association avec un imageur monochrome aboutit à la formation d'imageurs multispectrauxà 4,9 ou 16 bandes (figure 1).

Sur ce principe, SILIOS Technologies a ainsi développé les caméras multispectrales CMS (figure 2). Ces caméras sont basées sur un imageur CMOS de 1,3 Mpixels $(1280 \times 1024)$. Elles couvrent chacune un domaine spectral d'environ $300 \mathrm{~nm}$, discrétisé en 8 bandes spectrales ( 8 «couleurs») et 1 densité neutre ou pixel noir et blanc. Les différents domaines spectraux adressés sont les suivants:

- CMS-C: 430-700 nm,

- CMS-V: 550-830 nm,

- CMS-S: 650- $930 \mathrm{~nm}$.
Les caractéristiques des CMS sont données au tablean 1. La grande compacité $\left(52 \times 52 \times 22 \mathrm{~mm}^{3}\right)$ et le très faible point $(<60 \mathrm{~g})$ de ces solutions en font des instruments très adaptés pour les applications embarquées. La simplicité de leur architecture optique est également un avantage en termes de robustesse et de fiabilité.

La transmission spectrale de chaque pixel du macropixel des filtres présente une largeur de bande à mi-hauteur (FWHM) de l'ordre de 20 à $40 \mathrm{~nm}$. Ce type de résonnance à faible facteur de qualité $(\mathrm{Q})$ présente l'avantage d'une faible sensibilité à l'incidence angulaire. Ce point est particulièrement important pour une utilisation des filtres dans le domaine de l'imagerie car il permet de limiter l'influence de l'ouverture de l'objectif utilisé. D'autre part, cette largeur de bande confere au capteur multispectral une sensibilité suffisante pour la majorité des applications. La mesure du rendement quantique QE de l'imageur CMS-C par exemple met en évidence des valeurs pics comprises entre $20 \%$ et $35 \%$ (figure 3 ).

En termes d'utilisation, à partir de chaque image brute enregistrée par l'imageur multispectral, il est possible d'extraire les 9 sous-images spectrales (8 images «couleurs» + 1 image monochrome). La résolution de ces sous-images est de $426 \times 339$. De la même manière, un spectre à 8 bandes peut être extrait de chaque macro-point de l'image brute (figure 4). À partir de ces données, l'utilisateur est en mesure de développer le traitement d'image adapté à son application. La figure 5 présente une image retraitée issue d'une caméra CMS-V (550-830 nm). Le traitement mis en œuvre met en évidence par une coloration rouge, tous les objets de la scène présentant la signature spectrale de la chlorophylle.

L'intégration du filtre pixélisé multispectral peut être réalisée de deux manières. Soit par intégration monolithique (le filtre est fabriqué à la surface du capteur), soit par intégration hybride (le filtre est réalisé sur un substrat indépendant, puis reporté sur l'imageur et fixé). L'approche monolithique n'a d'intérêt que pour les très grands volumes de production. Elle ne tirera efficacement parti des méthodes de production collectives qu'au-delà de centaines de milliers de pièces ou

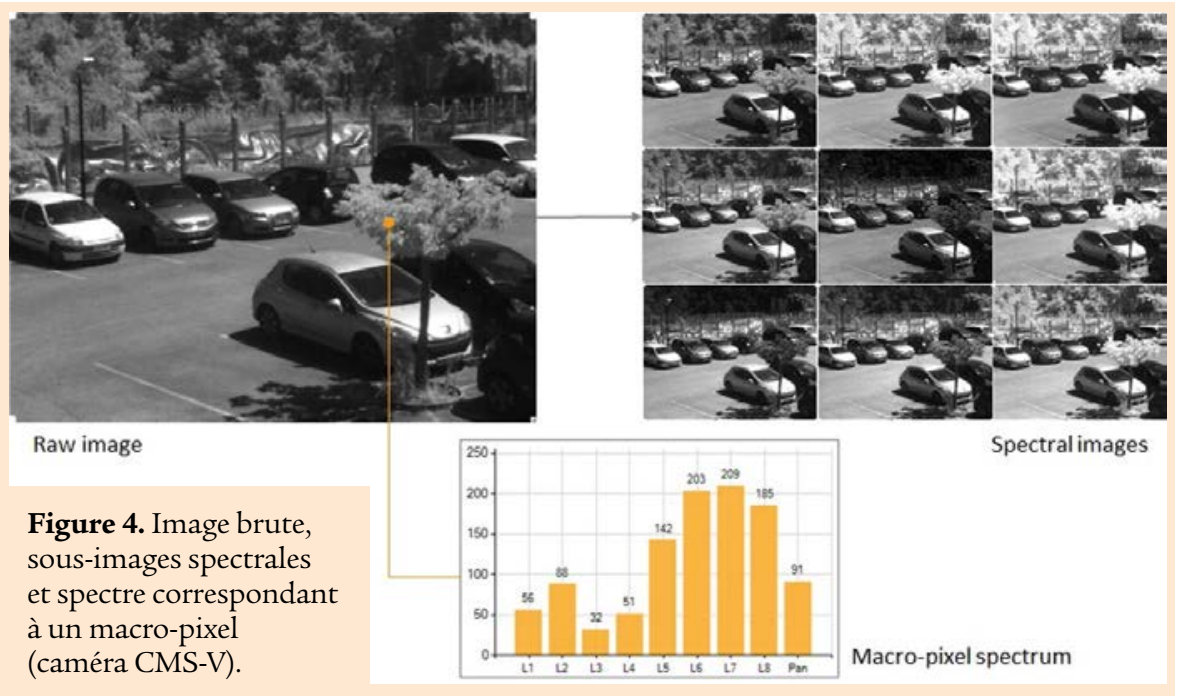




\begin{tabular}{|ll|}
\hline CAMERA SPECIFICATIONS & \\
\hline Optical Interface & CS-mount (C-mount compatible with additional C-ring) \\
\hline Resolution (raw picture) & $1280(\mathrm{H}) \times 1024(\mathrm{~V})$ \\
\hline Resolution (spectral pictures) & $426(\mathrm{H}) \times 339(\mathrm{~V})$ \\
\hline Maximum frame rate & $60 \mathrm{~Hz}$ \\
\hline Exposure time range & $10 \mu \mathrm{s} \mathrm{to} 2 \mathrm{~s}$ \\
\hline ADC & 10 -bit \\
\hline Camera control & USB 3.0 \\
\hline Digital output & 10 -bit USB 3.0 \\
\hline Power supply & USB 3.0 \\
\hline Dimension (W $\times \mathrm{H} \times \mathrm{L}$ ) & $52 \times 52 \times 22 \mathrm{~mm}^{3}$ \\
\hline Weight camera head & $59 \mathrm{~g}$ \\
\hline
\end{tabular}

Tableau 1. Caractéristiques principales des caméras CMS.

même de millions de pièces. Elle n'est économiquement pas adaptée aux productions de plus faibles volumes. Cette approche monolithique est développée par exemple par l'Institut IMEC en Belgique ou par l'entreprise Pixelteq aux États-Unis. Le premier propose deux versions de capteurs multispectraux de type Bayer à 16 ou 25 bandes dans le domaine visible ou proche infra-rouge dont la largeur spectrale FWHM est de l'ordre de 5 à $15 \mathrm{~nm}$, offrant ainsi une bonne finesse spectrale mais limitant aussi la sensibilité du capteur multispectral par bande. Le second utilise une technologie de structuration des dépôts (procédé lift-off) plus polyvalente en termes de bande spectrale adressée ou de gabarit de filtre, mais limitée à environ 20 microns en termes de taille minimum de pixel.

L'approche hybride développée par SILIOS Technologies permet d'adresser des volumes plus modestes (depuis quelques pièces à quelques milliers ou dizaines de milliers de pièces). Elle autorise facilement et avec des coûts réduits l'adaptation de la configuration spectrale du filtre à n'importe quel imageur du commerce, ce pour s'adapter aux multiples besoins du domaine de l'imagerie multispectrale. Enfin, l'approche hybride permet de conserver les microlentilles de l'imageur ce qui n'est pas possible en monolithique pour des raisons d'incidences trop importantes sur le filtre en sortie de microlentille. La présence de microlentilles permet de gagner $50 \%$ de flux supplémentaire sur chaque pixel, ce qui améliore la sensibilité de la caméra.

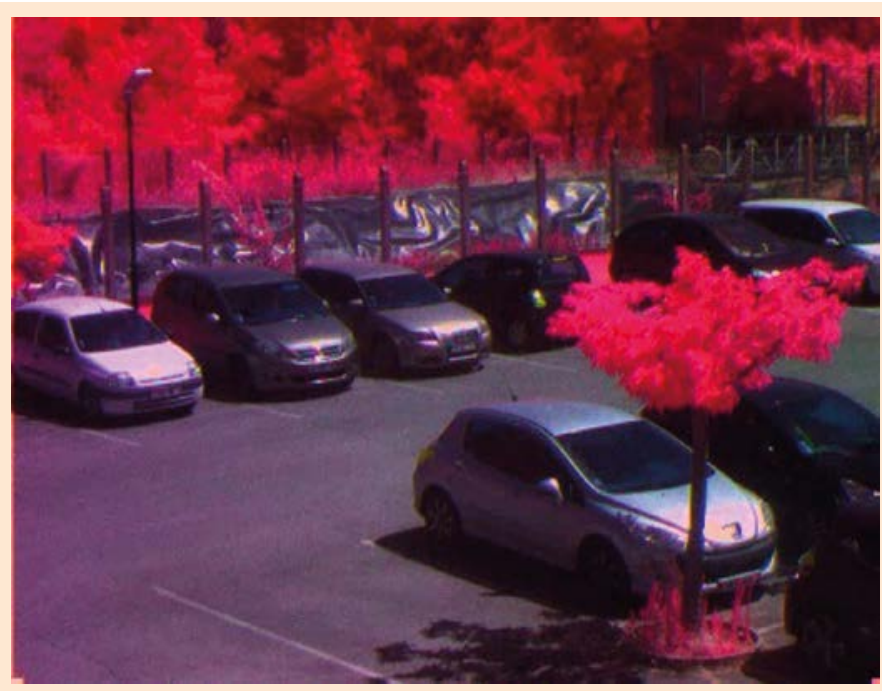

Pour finir, notons que le phénomène de diaphonie, c'est-àdire la pollution du signal d'un pixel par les signaux des pixels filtres voisins, existe dans ce type de systèmes multispectraux. En effet, l'inclinaison des rayons lumineux incidents sur le filtre en conduit une partie en sortie du filtre à impacter les pixels voisins. Ce phénomène est visible sur la figure 3 représentant le rendement quantique. On observe, pour chaque réponse spectrale des pixels, un certain niveau de signal en dehors de la bande d'intérêt. SILIOS a développé une méthode de correction de la diaphonie pour chaque pixel par application d'une combinaison linéaire des signaux des pixels voisins. Le résultat, dénommé «QE équivalent corrigé de la diaphonie», est présenté à la figure 6 . On constate que la réjection des bandes est ainsi considérablement améliorée. L'application de cette méthode de correction de la diaphonie permet d'accéder à des images spectrales et à des spectres bien plus contrastés. Le lecteur intéressé par cette méthode et ses résultats pourra se reporter à la note d'application disponible à l'adresse suivante: http://www.silios.com/cms-cameras (en bas de page).

\section{Conclusion}

L'ajout sur un imageur monochrome d'un filtre pixélisé multispectral agissant comme une matrice de Bayer customisée est une solution particulièrement intéressante pour beaucoup d'applications en imagerie multispectrale. Le cœur du système multispectral est ainsi fortement intégré. Il se réduit à un seul composant: le capteur multispectrale. Tous les avantages de la forte intégration suivent: compacité, légèreté, fiabilité, robustesse, bas coût et simplicité d'intégration dans les systèmes. Le capteur multispectral peut être intégré tout aussi simplement qu'un imageur standard. Ces solutions attirent un nombre croissant de secteurs d'applications vers l'imagerie multispectrale. L'exploitation des possibilités logicielles pour le traitement de la donnée multispectrale commence déjà à ouvrir le champ à des applications nouvelles que ne permettait pas l'imagerie standard. Les applications embarquées et l'Internet des objets en particulier vont très rapidement démultiplier les usages.

$\triangleleft$ Figure 5. Image en fausses couleurs mettant en évidence la signature chlorophyllienne.

$\nabla$ Figure 6. QE équivalent corrigé de la diaphonie (imageur multispectral CMS-C).

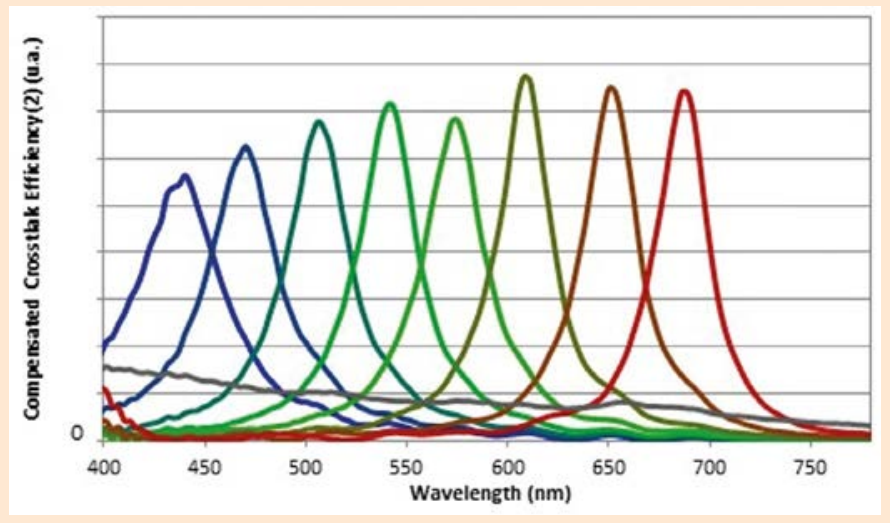

